\title{
FILIÈRES MILITANTES ET FIXATION DES PRIX : UNE ÉCONOMIE QUI S'INVENTE DANS UN CIRCUIT COURT D'IMPORTATION DE CAFÉ DU CHIAPAS
}

\author{
Mehdi Bouzouina, Juliette Rouchier \\ in Dominique Paturel et al., Le droit à l'alimentation durable en démocratie
}

Champ social | «Acteurs sociaux »

2020 | pages 215 à 224

ISBN 9791034605958

DOI 10.3917/chaso.patur.2020.01.0215

Article disponible en ligne à l'adresse :

https://www.cairn.info/le-droit-a-l-alimentation-durable-en-

democratie---page-215.htm

Distribution électronique Cairn.info pour Champ social.

(C) Champ social. Tous droits réservés pour tous pays.

La reproduction ou représentation de cet article, notamment par photocopie, n'est autorisée que dans les limites des conditions générales d'utilisation du site ou, le cas échéant, des conditions générales de la licence souscrite par votre établissement. Toute autre reproduction ou représentation, en tout ou partie, sous quelque forme et de quelque manière que ce soit, est interdite sauf accord préalable et écrit de l'éditeur, en dehors des cas prévus par la législation en vigueur en France. Il est précisé que son stockage dans une base de données est également interdit. 


\section{Filières militantes et fixation des prix : une économie qui s'invente dans un circuit court d'importation de café du Chiapas}

Mehdi Bouzouina \& Juliette Rouchier

Mutvitz34 est une association dont le but est l'importation et la vente directe de café issu des plantations des zapatistes au Mexique, ainsi que la dissémination d'informations sur le mouvement, à travers des actions de communication. Association à but non lucratif, elle s'affiche comme militante et le lien marchand tissé autour du café s'inscrit dans une démarche globale au mouvement zapatiste. Pour établir ce lien marchand, elle se positionne sur une logique de fixation des prix qui rappelle les AMAP, dans la mesure où les acheteurs se disent prêts à accepter la demande faite par les vendeurs du produit brut, sans négociation, et de partager les risques. Au final, les prix d'achat aux producteurs sont suffisants de leur point de vue, et le prix de la livre de café au consommateur est comparable à des prix de café bio et équitable dans le commerce, pas tellement plus cher que le haut de gamme de supermarché.

Cette fixation des prix qui ne passe pas par le marché concurrentiel est devenue assez fréquente dans les dernières années, mais concerne plus largement des circuits d'alimentation locaux. En effet, face à un produit transformé et importé sur de grandes distances comme le café, l'infrastructure pour importer, torréfier et distribuer le café est très importante, et repose finalement sur un bénévolat bien organisé et spécialisé, qui rend le modèle économique difficile à généraliser largement.

Le chapitre se propose de décrire l'association et son travail avec quelques détails. Ensuite une discussion sur la question de 
l'établissement du prix sera présentée, où sera discutée rapidement la notion de prix juste. Enfin, la liste des éléments nécessaires et parfois problématiques à l'organisation de ces importations sera listée, pour conclure sur la complexité de généraliser ce modèle économique, dont le développement dans son histoire spécifique est ici plutôt convaincant.

\section{MutVitz34 : bistoire et activité}

Créée en 2009, l'association Mutvitz34 est localisée dans le département de l'Hérault, et son but est de soutenir les zapatistes, au travers notamment de la vente en chaîne courte du café du Chiapas. Elle est née par essaimage au sein de réseaux militants qui soutiennent le mouvement zapatiste. En effet, les premières associations ayant créé le lien étaient celles de Toulouse et du Tarn, qui ont 20 ans. Dans le Sud de la France, elles travaillent ainsi en un large collectif, et se sont réparti certaines tâches liées à l'importation, ce qui sera décrit plus loin. Cette dynamique s'inscrit dans un contexte international de création de liens commerciaux directs avec le Chiapas, qui s'observe en Europe (Italie, Allemagne) et aux EtatsUnis, suivant des organisations un peu similaires.

L'association constitue (entre autres choses) un groupement d'achat, et réalise ainsi certaines étapes d'intermédiation entre les membres qui commandent et la coopérative agricole zapatiste (appelée Yachil) de la zone du Chiapas. La vente du café est proposée par paquets de $500 \mathrm{~g}$, importés sous forme de café vert, puis torréfié en France. L'achat et l'importation du café sont gérés à travers le réseau entier d'associations du Sud : les échanges s'effectuent par téléphone ou par mail et la négociation des prix est réduite au minimum puisque la coopérative fixe unilatéralement le prix. L'association du Tarn a réalisé le travail administratif pour l'importation durant plusieurs années, mais depuis 3 ans la " charge " de travail est tournante, Toulouse l'a fait durant 3 ans c'est maintenant au tour de MutVitz34. La torréfaction est externalisée, et le torréfacteur rémunéré en fonction des volumes traités, car les associations n'ont pas intérêt à faire l'investissement d'une machine au vu des besoins. Le coût de revient d'un paquet de $500 \mathrm{~g}$, pour l'association, est d'environ 5 euros, et il est proposé à 7 euros 50 pour dégager un surplus à envoyer comme don au Chiapas, ce qui représente donc $1 / 3$ de la somme totale. 
Tout comme on peut trouver dans le système des AMAPs (Lamine), l'engagement d'achat des consommateurs est fort. Les acheteurs paient $80 \%$ du prix du café à l'avance, soit dès le moment que le prix est fixé par la coopérative, au moment de la récolte, décembre. Ce paiement se fait en début d'année, et les $20 \%$ restants sont versés au départ du bateau du Mexique. Ceci peut ressembler à une façon de partager les risques, comme pour les AMAP : même si jamais il n'y a eu d'impossibilité pour les zapatistes à fournir la totalité des stocks demandés ni de perte lors du transport, le risque est accepté intégralement par les membres de l'association, qui peuvent tout perdre. Ce sont 250 sacs qui arrivent dans un container pour toutes les associations du Sud, chaque sac contenant $69 \mathrm{~kg}$ de café vert qui diminue à $55 \mathrm{~kg}$ une fois torréfié. Comme le container est plein, il serait assez compliqué d'augmenter aujourd'hui la quantité importée.

L'association oscille entre 150 à 200 adhérents, et les seuls départs ont été dus à des déménagements. Le recrutement s'est fait en général par le bouche à oreille, au sein de milieux associatifs déjà axés sur l'alimentation (AMAP), à travers des articles dans la presse, ou lors d'événements où des informations sont diffusées sur les zapatistes du Chiapas. Il y a environ 50 membres véritablement actifs, qui font à la fois de la diffusion d'information, du stockage de café dans des espaces privés, du transport, et organisent la torréfaction et la répartition en sachets standards. Le café n'est pas forcément distribué intégralement en une fois (la distribution peut être étalée dans le temps, tous les deux mois par exemple).

Le port de départ est Vera Cruz, et après être arrivé par Fos sur Mer, le café débarque depuis deux ans à Bordeaux. Le départ de Vera Cruz, port contrôle par une entreprise Bolloré, impose un grand aléas dans les coûts. En effet, les prix ont été multipliés par 2, puis 3, au cours du temps. Il peut arriver également que des interventions institutionnelles gênent le processus : le gouvernement mexicain, opposé aux zapatistes, a bloqué le café et imposé une amende pour qu'il puisse partir de Vera Cruz; en France la douane a estimé également que les quantités importées étaient trop importantes pour une association non lucrative, il y a eu un contrôle fiscal qui a résulté en un redressement. Le processus d'importation n'a donc pas de statut parfaitement clair et se trouve un peu en marge des normes commerciales, et a intérêt à osciller entre les filières habituelles, en évitant de se rendre trop visible. Les paiements à la coopérative qui se font sur un compte en banque. Le fond collecté en soutien direct 
aux activités des zapatistes est inclus dans le prix (2 euros 50 par paquet, soit 1/3) est é reversés par d'autres biais. La chaîne de transmission oscille donc entre des normes légales strictes et des formes de marginalité, ce qui crée un risque assez important pour les responsables mais permet également une certaine inventivité des pratiques. En outre, il y a très peu de fond de roulement pour l'adaptation à des aléas dans le processus : il n'y a pas de trésorerie spécifique à la résolution de problèmes comme ceux décrits au-dessus, ce qui ajoute une vulnérabilité assumée.

\section{Fixer des prix « justes »}

L'association s'inscrit donc dans un ensemble d'associations militantes qui visent à rétribuer justement les agriculteurs lors de l'achat du produit brut, afin de soutenir le travail de paysan. L'exemple le plus connu de nos jours sont les AMAPs, pour lesquelles la fixation des prix est également lieu d'une discussion intense autour de la valeur des biens et de la solidarité. Comme dans toutes les situations de ce type, la question de la fixation du prix juste est soumise à des discussions démocratiques - c'est en AG qu'est décidé dans ce cas les tarifs pour l'acheteur final. Mais comme le montrent de nombreuses discussions sur le sujet, un prix « juste » n'existe pas en soi. On peut s'approcher par contre d'un prix satisfaisant, ou acceptable, pour tous.

La discussion autour du prix juste est aussi ancienne que les premiers écrits sur les prix, et a été particulièrement importante au Moyen-Age, lorsque les marchés avaient un rôle de régulation des prix pour éviter la spéculation, et donc protéger les pauvres de la famine (Arnoux, 2012). D'intéressants articles scientifiques montrent combien cette discussion est réapparue sans cesse alors que la pensée économique évoluait (pour des bilans récents sur les produits alimentaires : Guillotreau, 2013 ; Prévost, 2012).

De la façon la plus simple et quasi définitionnelle, la fixation du prix induit ce que la théorie des jeux peut appeler « un jeu à somme nulle " entre deux individus : à chaque fois que le prix augmente, le vendeur est gagnant et l'acheteur perdant; à chaque fois que le prix baisse, le vendeur est perdant et l'acheteur gagnant. En toute logique on ne peut pas gagner en même temps.

Si l'on prend une vision un peu plus subtile, souvent utilisée pour faire les expériences en économie, on peut dire que l'acheteur 
et le vendeur ont chacun une limite (maximum et minimum) de prix d'achat ou de vente. Si le produit est vendu au prix minimum imaginable par le vendeur, tandis que l'acheteur fait une excellente affaire car il aurait pu acheter plus cher : alors l'acheteur fait un surplus, mais pas le vendeur. A l'inverse, si l'acheteur prend le produit à la limite maximum possible, et que le vendeur a un coût de revient beaucoup plus bas que ce prix, alors le vendeur fait un surplus mais l'acheteur aucun. On peut alors considérer qu'une transaction réussie (juste) partage le surplus entre le vendeur et l'acheteur de façon équitable.

On appelle "pouvoir de marché " la pression que peut mettre un des acteurs sur l'autre (Guerrien, 1996). En particulier, si des acheteurs peu nombreux (oligopsone) peuvent faire jouer la concurrence entre des vendeurs, ils réussissent à réduire le surplus du vendeur au maximum. A l'inverse, s'il y a beaucoup d'acheteurs et peu de vendeurs (oligopole), ceux-ci peuvent choisir leur surplus sans laisser de choix aux acheteurs, qui sont obligés de prendre ce qui est proposé. Entre les deux extrêmes, le monopsone et le monopole, toutes les situations peuvent exister et paraitront plus ou moins juste ou plus ou moins « acceptable " (Guillotreau, 2013).

Bien sûr, la question de ce que veut dire l'équité peut alors être $-219$ discutée. Par exemple : faut-il partager le surplus à part égale entre les deux (si on peut approximer les coûts et les alternatives pour chaque) ou faut-il que celui qui a le plus grand besoin soit aidé, quitte à ce qu'un des acteurs sacrifie un peu de son surplus au profit de l'autre? C'est en général la vision que développent ceux qui demandent un "prix juste " à l'heure actuelle, et qui mobilisent ce concept dans des contextes militants. Les associations se forment explicitement pour donner le plus large surplus possible au producteur, tout en restant dans un prix acceptable pour le consommateur. Quand on parle d'alimentation, ce prix juste est pensé en comparaison d'un prix injuste, qui est commun pour le monde agricole, contraint de vendre à très bas prix les matières premières, où des intermédiaires font des marges importantes et où les acheteurs finaux paient très peu cher leur alimentation (comparativement à d'autres périodes historiques). Les AMAP ont mis en place cette idée du prix juste sans réellement travailler originellement sur la fixation des prix : les prix d'achat étaient alignés sur le marché de qualité équivalente, mais c'est le paysan qui touchait l'intégralité du prix lors de la transaction, sans qu'un intermédiaire 
ne soit rétribué : cela faisait un prix très correct. Malgré un travail régulier depuis la création des AMAP pour ajuster le prix aux réels besoins annuels du paysan, et non s'aligner sur le prix du marché, la construction du juste prix n'est pas vraiment réalisée à ce jour (Rouchier, Lamine, 2016). Le cas de Mutvitz34 est un peu différent.

Le prix final du café est fabriqué comme somme des coûts intermédiaires tout au long de la chaine. Les coûts comptés sont : l'achat du café vert, le transport, l'importation, la torréfaction. Les trois derniers postes sont incompressibles et l'association ne peut en rien les décider. Le prix du café vert est fixé quant à lui d'une façon qui est considérée comme juste, car c'est la coopérative Yachil qui fixe le montant, et les acheteurs des pays riches acceptent de façon unilatérale l'offre qui est faite. Un second niveau de justice est néanmoins exprimé dans la fixation de ce prix, par les zapatistes euxmêmes. En effet, ils considèrent qu'ils sont obligés de s'auto-limiter dans leurs exigences financières, car les producteurs qui vivent autour de leur coopérative n'ont pas un choix aussi grand qu'eux, dans la mesure où ils n'ont pas accès à une consommation militante, et doivent s'aligner sur les prix très bas qui leur sont imposés par leurs intermédiaires. Tout en se situant dans la fourchette haute pour les

220 - cafés de ce type, le prix du café vert est donc conservé suffisamment bas pour ne pas créer trop d'injustice visible sur le marché local. Mutvitz34 s'est dotée en parallèle du moyen de collecter et redistribuer une part des achats, de façon plus informelle, pour compenser la limite que se posent les zapatistes, tout en restant dans les limites acceptables de prix pour les acheteurs militants.

\section{Une intermédiation militante}

Tout comme le système des AMAP, le groupement d'achat Mutvitz34 peut être considérée comme une intermédiation militante, qui differe de l'intermédiaire classique en ce qu'elle crée des liens marchands directs entre vendeur et acheteur, sans être en tant que telle propriétaire du bien ou de l'argent qui circulent d'un côté à l'autre de l'océan. Pour autant, on a vu qu'il existe une réelle complexité dans le processus d'échange, et qu'il est lié à la fois à la distance, au statut politique de la coopérative à laquelle le café est achetée, et au fait que le café est un produit nécessairement transformé. Dans ce dispositif marchand complexe, l'association prend en charge (ou mutualise avec certaines autres associations, comme dit au-dessus) un certain nombre de tâches nécessaire : 
- En premier lieu la discussion des prix en amont : même si dans ce cadre la négociation est réduite à une proposition unilatérale des offreurs, il a fallu qu'un contact soit créé dans ces termes il y a vingt ans, et tous les ans il faut actualiser par un appel les tarifs attendus.

- En sus de la création initiale de lien avec Yachil, la coopérative, la mise en place de l'organisation a nécessité une coordination forte entre des personnes attirées par l'idée de cette vente directe, et une communication initiale importante - surtout remise dans le contexte qui correspond à la création des AMAP, tandis que les groupements d'achat n'étaient pas encore très connus en France.

- À partir de cette connaissance, il faut collecter les contributions des membres de l'association ( $80 \%$ du prix final), ce qui permet de payer en avance le café vert.

- L'association fait également un travail de communication, marginalement pour attirer de nouveaux adhérents à l'heure actuelle car le nombre est suffisant, ou décrire à ceux qui sont déjà dans l'association les évolutions dans les initiatives des zapatistes, ainsi que la situation du café au cours du temps.

- La gestion financière est assurée par des membres de l'association.

- La gestion administrative de l'importation et du transport international est mutualisée entre toutes les associations du Sud, de façon tournante, ce qui permet de n'avoir qu'un interlocuteur pour gérer le container.

- Une gestion logistique est également mise en place en France, qui implique le transport depuis le port, la relation au torréfacteur et l'organisation des transports et répartition, le stockage dans des garages personnels, et la distribution auprès des membres.

- La gestion des crises ponctuelles diverses, comme la résolution du blocage du stock à Vera Cruz en $2 . .$. ?? par le gouvernement mexicain, et le suivi du contrôle fiscal et le paiement d'une TVA plus importante que prévu.

On peut constater que l'infrastructure est beaucoup plus importante que pour des groupements d'achats locaux comme les AMAPs et comportent des aléas encore plus grands du fait de la longueur physique de la chaine et les étapes de l'importation. En particulier on peut être impressionné par l'ampleur de ce qui relève de la prise en charge des coûts de transaction et des coûts de 
logistique par des bénévoles : le fait que cette part est gratuite en argent, bien que non gratuite en temps, permet de conserver le prix final dans une valeur acceptable pour les acheteurs.

La théorie des coûts de transaction, qui est née dans les années 30 suite à une intuition de Coase (1937), pense en effet l'interaction marchande comme plus coûteuse globalement que le simple prix d'échange. Ici, tout ce qui concerne la création du lien marchand pérenne et confiant, la mise en place du protocole d'échange, ainsi que la création d'une demande (à travers la sensibilisation des consommateurs devenus militants) peuvent être vus comme des coûts de transaction. On peut voir que leur prise en charge a été importante au moment de la création du lien marchand et qu'ils sont ensuite limités une fois que l'organisation a été mise en place, car ils relèvent d'une routine relationnelle. En outre, ces coûts (en temps pour préparer l'échange, attirer de nouveaux participants, ou en déplacement dans le Chiapas pour les premiers qui ont créé ces associations) ne sont pas dédiés qu’à la mise en place de la filière de café mais également à la constitution d'un collectif militant. De plus, contrairement aux AMAPs, qui imposent des rencontres hebdomadaires ce qui fait que ces coûts de transaction sont parfois 222 - perçus comme trop élevés (Olivier et Coquart, 2010), Mutvitz34 ne mobilise les acheteurs que lors de quelques distributions annuelles et fonctionne dans une routine et une mutualisation d'activité qui ne rend pas ces coûts trop élevés pour les bénévoles.

On peut s'interroger sur l'ampleur des coûts logistiques bénévoles, et le rôle d'assurance qu'acceptent de jouer les acheteurs finaux. Comparativement aux AMAPs, les problèmes potentiels se sont déjà réalisés dans un large éventail, qui ne relèvent pas seulement de soucis productifs mais aussi d'empêchements qui peuvent être liées à des oppositions politiques aux intentions même de la chaîne, autant qu'à des augmentations de tarif énormes des droits dont l'accès dépend d'un monopole. En outre, un événement qui ne s'est pas réalisé mais qui peut faire partie des risques dans cette chaîne est liée au stockage non professionnel, dont on imagine qu'il peut occasionner la dégradation des produits, voire des ennuis sanitaires. De ceci on peut conclure que cette logistique totalement bénévole, complexe et nécessitant un investissement des participants vraiment important, peut être vue comme la force actualisée et la faiblesse potentielle de ce modèle d'échange. 


\section{Conclusion}

Née dans la période du renouveau des circuits courts alimentaires, l'expérience de circuit court alimentaire autour du café zapatiste est à ce jour une réussite attestée par le nombre d'adhérents, la continuité des échanges, ainsi que par l'implication importante de participants réellement actifs dans le circuit. C'est à peu près $1 / 3$ des membres qui s'impliquent dans les actions de sensibilisation et de logistique qui sont le gros de l'action bénévole de l'association. Ce ratio est plutôt élevé pour ce type de circuit (Mundler, Rouchier, 2016), ce qui marque peut-être le fait que l'engagement politique sous-jacent est plus important pour les membres que dans d'autres circuits. On peut une fois de plus noter que bien que ne payant pas de leur personne pour faire exister l'institution d'échange, les acheteurs peu impliqués - ceux qui ne font qu'acheter - sont nécessaires, car ils acceptent les risques de la chaîne et permettent d'atteindre une demande suffisante pour importer des quantités telles que la mutualisation des tâches soit performante, ce qui réduit de façon relative les coûts de transaction.

La question de la possibilité l'" essaimage " (généralisation, diffusion) des initiatives de circuits courts, est classique. Ici, Mutvitz34 est déjà le résultat d'un essaimage au sein d'un réseau plus ancien : cette diffusion a eu lieu et le développement a atteint son potentiel maximum, qui correspond à un container entier d'importation pour le Sud de la France. On peut donc se poser la question d'une influence plus générale qui se traduirait par la mise en place d'un circuit équivalent dans d'autres régions françaises ou, de façon plus intéressante, sur d'autres produits. On a noté que la plupart des coûts se concentraient dans l'investissement initial que représente la création de la chaîne (création d'un lien à longue distance, apprentissage des règles administratives de transport et importation, mise en place des échanges financiers, création d'une demande) et que le risque est très important pour les participants. Il semble logique qu'il soit impossible qu'une telle organisation se déploie sans une implication d'ordre politique très forte, ce qui réduit l'ensemble des produits d'échange envisageable. En outre, le stockage bénévole fait penser que la même organisation ne pourrait s'appliquer par exemple qu'à des produits très peu périssables (et de fait, ces échanges longue distance existent surtout pour les agrumes). Exemple par sa réussite, Mutvitz34 et son réseau, ne sont pas forcément si faciles à imiter. 


\section{BIBLIOGRAPHIE}

Arnoux Mathieu, 2012, Le temps des laboureurs. Travail, ordre social et croissance en Europe (XIe-XIV siècle), Paris, Albin Michel ("L'évolution de l'humanité »).

Chiffoleau Yuna, 2019, Les circuits courts alimentaires. Entre marché et innovation sociale, Edition Eres.

Coase, Ronald H., The Nature of the Firm (1937). Economica (new series), Vol. 4, Issue 16, p. 386-405.

Guerrien Bernard, 1996, Dictionnaire d'analyse économique, La Découverte.

Guillotreau Patrice, 2013, Le juste prix des produits alimentaires : entre efficience des marchés et exigence de justice sociale, Economie rurale, mai-juin, pp 87-94.

Lamine Claire, 2017, Les AMAP: un nouveau pacte entre producteurs et consommateurs? éditions Yves Michel.

Mundler Patrick, Rouchier Juliette (éditeurs), 2016, Alimentation et proximités, Jeux d'acteurs et territoires, Educagri.

Prévost Benoit, 2012, Echanges alimentaires et juste prix. Un détour par l'histoire de la pensée économique pour alimenter un débat contemporain, L'homme et la société, 1, 183-184, pp 35-59.

Lamine Claire, Rouchier Juliette, 2016, D’une charte l'autre. Le processus de révision de la charte des AMAP comme indicateur d'une institution qui se renforce ? Revue de la régulation, 20, décembre,

Olivier Valérie, Coquart Dominique, 2010, Les AMAP : une alternative socio-

224 - économique pour des petits producteurs locaux ?, Economie rurale, 318-319, pp 20-36. 